\title{
A young perspective on an aging disease
}

\author{
Eli York
}

\section{University of British Columbia}

In 2012, the World Health Organization announced that dementia affected 35.6 million people worldwide, a number predicted to rise to 81.1 million by 2040 , making it a "public health priority." ${ }^{1}$ The associated economic burden is over $\$ 604$ billion US, largely due to costs of long term intensive care. ${ }^{1}$ In contrast, there are only five FDAapproved drugs for Alzheimer's disease (AD) treatment, which slow cognitive decline but do not provide a cure. ${ }^{2}$ Although there has been a surge of research on $A D$ and related dementias, our understanding of the disease - and potential cures - have not seen satisfactory advances. Why is there such a disparity between amount of research and number of scientific breakthroughs? One possibility is that, until recently, this field has been stuck in a narrow view of $A D$ mechanisms. As is a danger in all disciplines, main ideas potentiate and become the dogma, dictating how researchers design their experiments. In AD research, we see this in its intimate tie to amyloid- $\beta$ plaques and the use of genetic mouse models. Finally, the past bias is made clear by the promising developments of researchers who have approached $A D$ from fresh angles.

AD was first described in 1906 by Dr. Alois Alzheimer, who characterized it by the presence of neurofibrillary tangles and amyloid- $\beta$ plaques. ${ }^{3}$ To this day, AD is confirmed only at autopsy by the presence of plaques and tangles. ${ }^{4}$ Therefore, these features have become a hallmark of $A D$, and they are the focus of most research aims. From this sprang the amyloid hypothesis of $A D$, which is rich in scientific literature and which often targets clearance of plaques to treat dementia. ${ }^{5}$ In these experiments, amount of amyloid pathology is often used as a marker of treatment efficacy. However, plaques develop normally throughout aging brains, even in healthy individuals with no signs of cognitive impairment. ${ }^{6}$ In AD, there is evidence that brain areas with the highest plaque loading do not correlate with degree of neurodegeneration. ${ }^{4}$ Even once it was recognized that the
$A \beta_{42}$ oligomer is the more toxic form of the protein, research continued to be driven by studies targeting plaques, or that use them as an indicator for degree of dementia.

Another obstacle in $A D$ research is the lack of appropriate animal models. Mutations in genes that increase amyloid- $\beta$ plaques have become the classic AD mouse model. ${ }^{7}$ These mutations were identified in families with high prevalence of $A D$; however, genetically inherited $A D$ accounts for only $1-5 \%$ of total cases, while the majority are sporadic. ${ }^{8}$ Since mechanisms causing sporadic $A D$ are unknown, there is no appropriate mouse model to investigate treatment methods. Currently, the only factor clearly associated with sporadic $A D$ is age, and the average mouse lifespan is only two to three years. It is likely that the environment of a twoyear-old brain, even with genetic mutations, is very different from that of an 80 -year-old brain. These inequalities may explain the abundance of promising therapeutics for mice that do not hold up in clinical trials, as seen recently with Solanezumab (Eli Lily) and Bapineuzumab (Pfeizer/Johnson \& Johnson). Therefore, if we hope to treat sporadic AD in human patients, a more appropriate animal model will be needed.

Our knowledge on the roles of amyloid- $\beta$ plaques and neurofibrillary tangles will no doubt be invaluable to uncovering the secrets of AD. However, focusing too narrowly on one facet of a disease comes at the expense of overlooking critical factors or complex interactions between systems. AD truly develops over a lifetime, so focusing solely on aging-related properties risks missing potential initiators of the disease, as well as opportunities for early intervention. In fact, promising current research incorporates non-genetic risk factors such as lifestyle, traumatic brain injury, and the immune system. One recent paper has analyzed the effects of cellular oxidative stress as a potential risk for sporadic AD. ${ }^{9}$ Another group focuses on an excess of pro-inflammatory signaling in $A D$, and they has developed Etanercept, a TNF $\alpha$ inhibitor. ${ }^{10}$ This 
drug has reached clinical trials and is showing promising results in tests of cognitive performance, although its exact mechanism of action is not well understood. Clearly, recent unconventional research should be pursued and perhaps combined with other fields to create a multifactorial approach to a complex disease.

It is important to take our vast resource of information and approach the study of diseases from fresh directions to better understand how they can be prevented and cured. The future of scientific discovery is exciting as methods of imaging and analysis are being improved, providing clearer insight into both the process and time-course of disease progression. This discussion of $A D$ underscores the importance of innovative research that challenges dogmas in making scientific advancement possible. This is both our aspiration as researchers and our responsibility as a society.

\section{References}

1. World Health Organization, Alzheimer's Disease International. Dementia: A public health priority. (United Kingdom). 2012.

2. Alzheimer's Association. Alzheimer's Association National Office. 2013 [cited 2013 Feb 4]. Available from: http://www.alz.org/research/science/ alzheimers_disease_treatments.asp\#approved

3. Berrios G. Alzheimer's disease: A conceptual history. Int. J. Geriatr. Psychiatry. 1990; 5(6): 355-365.

4. Reitz C, Brayne C, Mayeux R. Epidemiology of AD. Nat Rev Neurol. 2011; 7(3): 137-152

5. Hardy J, Selkoe D. The amyloid hypothesis of Alzheimer's disease: Progress and problems on the road to therapeutics. Science. 2002; 297(5580): 353-356.

6. Josephs $\mathrm{K}$ et al. Beta-amyloid burden is not associated with rates of brain atrophy. Ann Neurol. 2008; 63(2): 204-212.

7. Hall A, Roberson E. Mouse models of Alzheimer's disease. Brain Res. Bull. 2012; 88(1): 3-12.

8. Fisher Center for Alzheimer's Research Foundation. New York: Fisher Center for Alzheimer's Research Foundation. 2012 [cited 2013 Mar 20]. Available from: http://www.alzinfo.org/07/about-alzheimers/genetic-risk-factors

9. Ramamoorthy $\mathrm{M}$ et al. Sporadic Alzheimer disease fibroblasts display an oxidative stress phenotype. Free Radical Biol. Med. 2012; 53(6): 1371-1380.

10. Tobinick E. Rapid improvement in verbal fluency and aphasia following perispinal etanercept in Alzheimer's disease. BMC Neurology. 2008; 8(27).

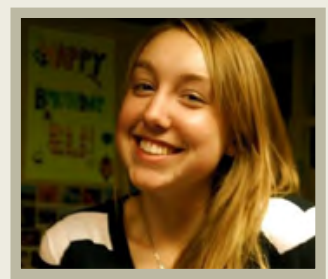

\section{Eli York}

Eli York is a first year Master's student in the Neuroscience program at the University of British Columbia. Her thesis project focuses on epigenetic regulation of aging in microglia, and how this process may contribute to sporadic, late-onset neurodegenerative diseases. Outside of the lab, Eli volunteers with the Alzheimer's Society of Canada in the Minds in Motion program, encouraging both mental and physical activity. 